\title{
Dynamique des populations fongiques et bactériennes de la rhizosphère des noisetiers truffiers III. Effet du régime hydrique sur la mycorhization et la microflore associée
}

\author{
M Mamoun *, JM Olivier \\ INRA, station de recherches sur les champignons, centre de recherches de Bordeaux, \\ 33883 Villenave-d'Ornon Cedex, France
}

(Reçu le 3 août 1989 ; accepté le 31 octobre 1989)

\begin{abstract}
Résumé - La colonisation par Tuber melanosporum de jeunes racines de noisetiers est fortement influencée par la quantité d'eau apportée quotidiennement. La présence et la multiplication d'espèces mycorhiziennes autres que la truffe s'accroissent avec la quantité d'eau, ce qui apporte de nouveaux éléments concernant le problème de la compétition dans les truffières irriguées. Enfin, la composition et la dynamique de la flore bactérienne de la mycorhizosphère, et tout particulièrement celles des types chélatants le fer, sont modifiées par des apports excessifs d'eau. Les essais confirment la résistance à la sécheresse de la mycorhize de Tuber et de l'écosystème associé.
\end{abstract}

potentiel hydrique / Tuber / mycorhization / compétition / Pseudomonas

Summary - Dynamics of fungal and bacterial populations of truffle-producing hazeinut trees. III. Effect of the soil water content on mycorrhization and the associated microflora. The volume of water supplied to mycorrhizal hazelnut trees (fig 2) induces variations in the composition and dynamic of the mycorrhizospheric bacteria (fig 3). The higher proportion of fluorescent Pseudomonas is observed in very humid conditions when the temperature is not a restricting factor (fig 4a). On the contrary, actinomycetes are strongly affected by water and very resistant to dryness (fig 4b). The iron chelating capacity of fluorescent Pseudomonas increases with soil water content (fig 5). Taxonomic studies (fig 7) have shown that, after a $150 \mathrm{~d}$ treatment, dryness and high irrigation are responsible for a decrease in $\mathrm{P}$ fluorescens levels. P putida populations increase with all treatments and especially with high irrigation. Intermediate taxon levels $\left(i_{1}\right.$ and $\left.i_{2}\right)$ remain low. Strong iron chelating capacities $(C M />32 p p m)$ are connected with to $\mathrm{P}$ putida and $\mathrm{P}$ fluorescens (fig 8). The incidence of irrigation on tree development and mycorrhization is observed. $A$ linear correlation between the surface and the length and width of the leaves of hazelnut trees was previously obtained (fig 1). Dryness has no effect on shoot development (fig 9b) but the sum of the leaf surfaces is correlated with the water supply (fig 9c). Root development and mycorrhization are evaluated as described in table 1 . The total mycorrhization level is the same in the 3 treatments but the Tuber to competitors ratio depends on the water supply (fig 10). No root development occurs in dry conditions but Tuber mycorrhiza are not affected. On the contrary high irrigation results in numerous young roots essentially colonized by competitors which also act as Tuber substitutes on older roots. A moderate irrigation induces a good root development, an expansion of the Tuber mycorrhization and very few competitors. The results indicate that $\mathrm{T}$ melanosporum and the associated ecosystem are well adaptated to dryness. The incidence of irrigation on the perenniallity of Tuber symbiosis and the fructification is discussed.

matric potential / Tuber / mycorrhization / competition / Pseudomonas

\section{INTRODUCTION}

Une étude microbiologique de la rhizosphère de noisetiers mycorhizés par Tuber melanosporum a mis en évidence une corrélation entre effectifs de
Pseudomonas fluorescents et teneur en eau du sol, au cours des saisons (Olivier, Mamoun, 1988). Les Pseudomonas isolés du rhizoplan de ces noisetiers représentent un pouvoir chélatant global vis-à-vis du fer nettement supérieur à celui détecté dans le sol nu (Mamoun, Olivier, 1989).

* Correspondance et tirés à part 
Les sidérophores produits par les champignons ectomycorhiziens (Szanislo et al, 1981) et par les Pseudomonas fluorescents (Hohnadel, Meyer, 1986 ; Scher, 1986) pourraient intervenir dans l'assimilation du fer par la plante (Römheld, 1987). Les sols de truffières sont chlorosants, et l'équilibre $\mathrm{Fe} \mathrm{II/Fe} \mathrm{III} \mathrm{varie} \mathrm{avec} \mathrm{le} \mathrm{potentiel}$ hydrique (Lindsay, 1974). Dans le cas de noisetiers truffiers, une compétition pour le fer entre Pseudomonas et Tuber a été envisagée comme hypothèse, en liaison avec le potentiel hydrique.

Dans un sol maraîcher calcaire, une augmentation du pF entraîne une baisse du pouvoir chélatant des Pseudomonas (Lemanceau et al, 1988). Des apports d'eau modérés sont favorables à la fructification de la truffe (Le Tacon et al, 1982) mais une irrigation excessive entraîne un arrêt de la production (Giraud, 1988). Aucune donnée, par contre, ne renseigne sur l'impact du régime hydrique sur l'évolution de la mycorhization. Pour préciser l'effet de l'irrigation, des noisetiers mycorhizés par $T$ melanosporum sont élevés en serre et soumis à 3 traitements hydriques recréant des conditions de sécheresse, de culture irriguée "normale», et d'irrigation forcée. L'évolution de la mycorhization est suivie (intensité, nature) ainsi que celle des populations de Pseudomonas fluorescents (effectifs, pouvoir chélatant vis-à-vis du fer, répartition taxonomique) et l'effet de l'irrigation sur le comportement relatif de ces organismes est discuté.

\section{MATÉRIEL ET MÉTHODES}

Les noisetiers ont été mycorhizés par la société Agritruffe avec Tuber melanosporum selon le procédé INRA-ANVAR. Ils sont âgés de 1 an au début de l'expérimentation. La terre est prélevée sur la truffière INRA de Dordogne où ont été faites les études microbiologiques citées précédemment. Elle est débarrassée des fragments de roche calcaire supérieurs à $3 \mathrm{~cm}$, et désinfectée par la chaleur ( 2 fois $1 \mathrm{~h}$ à $100^{\circ} \mathrm{C}$, à $24 \mathrm{~h}$ d'intervalle). Chaque pot reçoit une plante mycorhizée et $1,250 \mathrm{~kg}$ (MF) de terre désinfectée, soit environ $1 \mathrm{~kg}$ MS (humidité au moment de la plantation: $26,8 \pm 1,9 \%)$; MF désigne la matière fraîche et MS la matière sèche.

\section{Répartition des noisetiers par lots}

A la plantation, les développements aériens et racinaires des plantes présentant peu de variations d'un sujet à l'autre, les noisetiers sont triés de manière à avoir une répartition homogène de la mycorhization dans chaque lot. Deux critères sont retenus : l'intensité (échelle de 0 à 4 , cf tableau I) et la nature de la symbiose (identification au microscope photonique de prélèvements effectués dans l'ensemble du système racinaire). Trois lots de 24 noisetiers sont constitués ( 1 par régime hydrique), et chacun est subdivisé en 4 groupes de 6 .

\section{Régimes hydriques}

Ils sont déterminés d'après les relations entre teneur en eau du sol (terre fine) et effectifs de Pseudomonas

Tableau I. Échelles de notations.

\begin{tabular}{|c|c|c|c|c|}
\hline \multicolumn{3}{|c|}{ Échelles de mycorhization } & \multicolumn{2}{|c|}{ Échelles de développement racinaire } \\
\hline Note & Intensité & Spinules & Volume racinaire & Jeunes racines \\
\hline 0 & nulle & aucune & $\begin{array}{l}\text { très petit } \\
\text { (aucune progression } \\
\text { depuis la plantation) }\end{array}$ & aucune \\
\hline 1 & faible & $\begin{array}{l}\text { bien réparties mais } \\
\text { peu nombreuses ou } \\
\text { groupées à l'apex }\end{array}$ & $\begin{array}{l}\text { petit } \\
\text { (faible progression) }\end{array}$ & très peu, et courtes \\
\hline 2 & $\begin{array}{l}\text { moyenne et bien } \\
\text { répartie ou abondante } \\
\text { mais localisée }\end{array}$ & $\begin{array}{l}\text { assez nombreuses } \\
\text { et bien réparties }\end{array}$ & $\begin{array}{l}\text { moyen (bien ramifié } \\
\text { et quelques longues } \\
\text { racines) }\end{array}$ & $\begin{array}{l}\text { moyennement } \\
\text { ou peu nombreuses } \\
\text { mais longues }\end{array}$ \\
\hline 3 & $\begin{array}{l}\text { abondante et bien } \\
\text { répartie, mais pas } \\
\text { de glomérule }\end{array}$ & $\begin{array}{l}\text { nombreuses et } \\
\text { parfois ramifiées }\end{array}$ & $\begin{array}{l}\text { important } \\
\text { (longues racines très } \\
\text { ramifiées) }\end{array}$ & $\begin{array}{l}\text { abondantes et } \\
\text { longues }\end{array}$ \\
\hline 4 & id. 3, avec glomérules & & $\begin{array}{l}\text { très important } \\
\text { (remplit tout le pot) }\end{array}$ & $\begin{array}{l}\text { très abondantes, } \\
\text { très longues et bien } \\
\text { ramifiées }\end{array}$ \\
\hline
\end{tabular}


observés en truffière (Olivier, Mamoun, 1988). Des humidités de 21 et $31 \%$ dans la terre fine (correspondant à des apports de 160 à $250 \mathrm{~g}$ d'eau $/ \mathrm{kg}$ de sol sec) sont retenues pour simuler respectivement des conditions normales d'arrosage (lot $N$ ) et une irrigation forcée (lot F), selon les données de Giraud (1988). Une teneur en eau de $70 \mathrm{~g} / \mathrm{kg}$ de sol sec, soit une humidité d'environ $12 \%$, représente des conditions de sécheresse (lot S). Les quantités d'eau sont ajustées lors de la pesée journalière des pots. Les apports cumulés par mois sont exprimés en $\mathrm{mm}$ (ou $\mathrm{l} / \mathrm{m}^{2}$ ) comme en météorologie. Tous les plants sont soumis au traitement $\mathrm{N}$ pendant une période de $45 \mathrm{j}$ après la plantation. Ensuite, les 3 régimes hydriques sont appliqués, le temps 0 de l'expérience étant pris à ce stade.

\section{Analyses microbiologiques}

Chaque groupe de 6 noisetiers sert à tour de rôle pour les prélèvements de manière à laisser les populations microbiennes se stabiliser entre 2 prises d'échantillon. Les analyses sont effectuées à 0, 10, 30, 45, 105, 154 et $203 \mathrm{j}$ après le début des traitements hydriques, selon la technique décrite par Olivier et Mamoun (1988). Par date et par lot, 6 prélèvements (soit 1 par noisetier) sont traités séparément. Les échantillons sont constitués de fragments de racines courtes (environ $5 \mathrm{~cm}$ de long) portant des mycorhizes et de leur terre adhérente (environ $80 \mathrm{mg}$ MS). Après analyse, la terre de chaque échantillon est récupérée, déshydratée $24 \mathrm{~h}$ à $110^{\circ} \mathrm{C}$ et la matière sèche est déterminée. Les effectifs bactériens sont exprimés en logarithme du nombre d'unités formant une colonie par gramme de terre sèche $(\log$ CFU/g MS).

\section{Collection de Pseudomonas fluorescents}

Des isolats de Pseudomonas fluorescents sont prélevés aux temps 0,30 et $154 \mathrm{j}$, selon le protocole décrit par Mamoun et Olivier (1989). Les collections sont répertoriées dans le tableau II.

\section{Détermination du pouvoir chélatant vis-à-vis du fer}

Les capacités chélatantes des isolats sont évaluées selon la technique à l'hydroxy-8-quinoléine décrite par Lemanceau (1988) et utilisée par Mamoun et Olivier (1989). Les isolats sont incubés en présence de doses croissantes $(0,2,4,8,16,32$ et $64 \mathrm{ppm})$ d'hydroxy-8quinoléine et la concentration minimale inhibitrice (CMI) est déterminée (photomètre Titertek Multiscan

Tableau II. Collections de Pseudomonas fluorescents.

\begin{tabular}{cccc}
\hline $\begin{array}{l}\text { Date de prélèvement } \\
\text { (jours) }\end{array}$ & \multicolumn{3}{c}{ nombre d'isolats/traitement } \\
\cline { 2 - 4 } & $S$ & $N$ & $F$ \\
\hline 0 & - & 56 & - \\
30 & 42 & 48 & 51 \\
154 & 35 & 42 & 52 \\
\hline
\end{tabular}

MCC, $\lambda=620 \mathrm{~nm}$ ). Les résultats sont exprimés, pour chaque classe de pouvoir chélatant, en pourcentage des isolats $(\mathrm{PI})$ et en effectif $(\mathrm{E})$ calculé à partir de l'effectif total dénombré à la date d'isolement (Mamoun, Olivier, 1989).

\section{Taxonomie}

Les Pseudomonas fluorescents sont répartis à l'intérieur des espèces fluorescens et putida, et des 2 groupes intermédiaires définis selon Palleroni (1984). Pour cela, 2 aptitudes métaboliques, l'utilisation de tréhalose et la protéolyse, sont mises en évidence selon la technique utilisée par Mamoun et Olivier (1989). Les groupes intermédiaires sont nommés $i_{1}$ et $i_{2}$.

\section{Développement du système aérien des noisetiers}

Pour évaluer le développement aérien des noisetiers, plusieurs critères ont été choisis : le nombre de tiges, leurs longueurs cumulées, la longueur de la pousse de l'année de la tige principale, la surface foliaire de cette pousse de l'année, et la surface foliaire totale de chaque plante. Une corrélation linéaire entre la surface d'une feuille (S) et ses longueur (L) et largeur (I) a été déterminée préalablement (fig 1). L'équation obtenue est $S=0,73(L \times I)-0,27$, et le coefficient de régression $r^{2}=0,98$. La surface foliaire est ainsi déterminée par calcul d'après les mesures de longueur et largeur des feuilles. La nature du traitement hydrique ne modifie pas l'équation, permettant ainsi les comparaisons.

\section{Évolution du système racinaire et de la mycorhization}

Les contrôles sont effectués en fin d'expérimentation, soit 7 mois après le début des traitements hydriques.

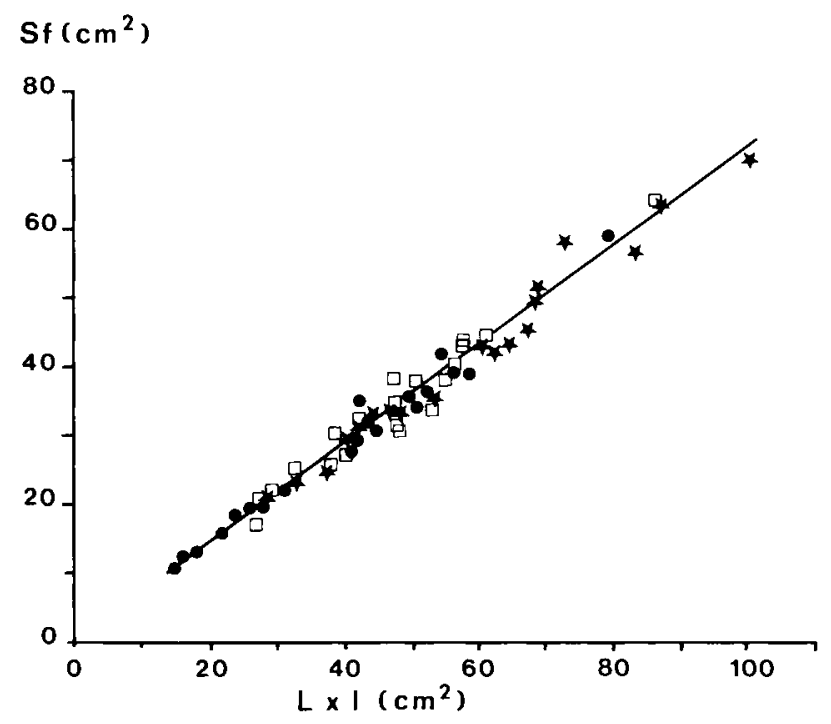

Fig. 1. Corrélation linéaire entre le produit longueur $x$ largeur $(L \times$ I) et la surface (Sf) chez la feuille de noisetier. $S$, $N$, DF. 
Le développement du système racinaire est évalué (échelle 0 à 4, cf tableau I), ainsi que la formation de jeunes racines (échelle 0 à 4 , cf tableau 1). La mycorhization (importance et nature) est déterminée selon la technique utilisée à la plantation. La distinction est faite entre mycorhization du vieux et du jeune système racinaire. Les spinules caractéristiques des mycorhizes de $T$ melanosporum (Cailleux, 1982) sont quantifiées (échelle 0 à 3 , cf tableau I).

\section{Représentations graphiques}

Les données rapportées sur les graphiques sont des moyennes accompagnées de leur écart type.

\section{RÉSULTATS}

\section{Régime hydrique}

Pour obtenir la teneur en eau fixée au début de l'expérience, les plants utilisés pour les prélèvements ont reçu, durant le mois précédant chaque analyse, les quantités d'eau indiquées fig 2 . Les valeurs très basses notées à $203 \mathrm{j}$ ont pour origine le ralentissement de l'activité hivernale des noisetiers. Entre 30 et $154 \mathrm{j}$, les irrigations sont de l'ordre de $20 \mathrm{~mm} / \mathrm{mois}$ pour le lot $\mathrm{S}$, et de $50 \mathrm{~mm} /$ mois pour le traitement $\mathrm{N}$. Elles sont plus fluctuantes, 80 à $125 \mathrm{~mm} / \mathrm{mois}$, pour le lot $\mathrm{F}$ proche des conditions de saturation.

\section{Analyses microbiologiques}

Les analyses microbiologiques montrent que, pour les traitements $S$ et $N$, les populations bac-

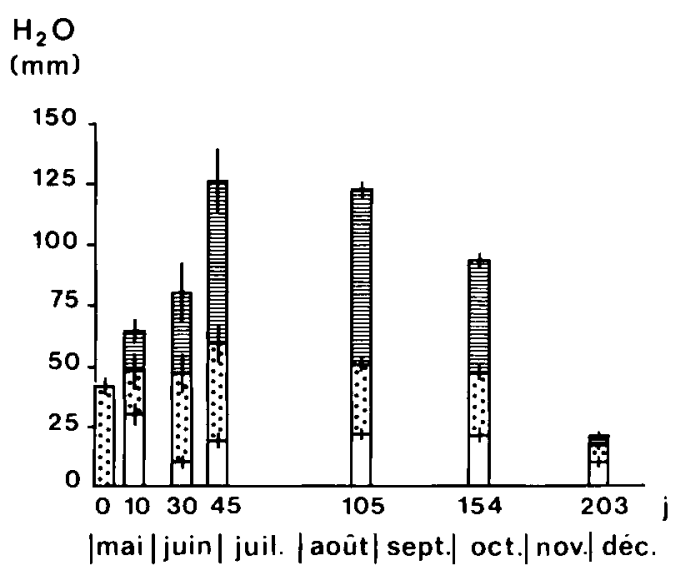

Fig. 2. Cumul des irrigations effectuées pendant le mois précédant les analyses microbiologiques. $\mathrm{j}=$ dates d'analyses (en jours après le début des traitements hydriques). $\square \mathrm{s}$, N, 冒 $\mathrm{F}$. tériennes totales sont à des niveaux proches et varient peu en cours d'expérimentation (autour de $1,4.10^{8} \mathrm{CFU} / \mathrm{g} \mathrm{MS}$ ). Par contre, les effectifs sont plus faibles dans le lot $F$, et la différence est significative à partir de $105 \mathrm{j}$ de traitement (fig 3a).

Les populations de bactéries non fluorescentes présentent la même dynamique que la flore totale, cependant la baisse d'effectifs dans le lot $F$ est beaucoup moins marquée et n'est, en général, pas significative (fig $3 b$ ).

Les niveaux de populations d'actinomycètes diminuent progressivement pendant $30 \mathrm{j}$ dans les 3 lots. Puis, pour les traitements $S$ et $N$, les effectifs augmentent pour se stabiliser, à partir de $105 \mathrm{j}$, autour du niveau observé au temps 0 $\left(4,9.10^{7} \mathrm{CFU} / \mathrm{g} \mathrm{MS}\right)$. Dans le lot F, ils baissent jusqu'au temps $45 \mathrm{j}$, puis se stabilisent autour de 2,0.10 $\mathrm{CFU} / \mathrm{g} \mathrm{MS}$ (fig 3c).

Les effectifs de Peudomonas fluorescents présents dans le lot $S$ diminuent progressivement
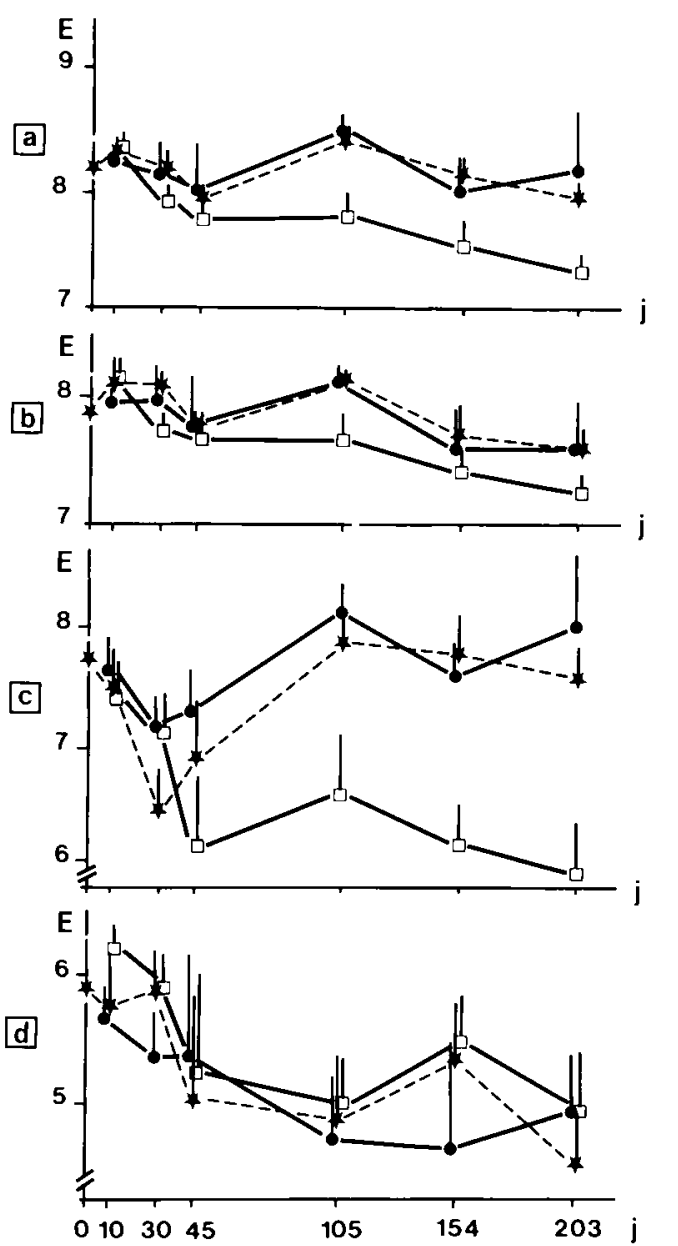

Fig 3. Dynamique des populations bactériennes en fonction du régime hydrique. $a$ flore totale, $b$ bactéries non fluorescentes [c actinomycètes, d] Pseudomonas fluorescents. $E=$ effectifs bactériens ( $\log C F U / g ~ M S), j=$ id fig 2; - $S$, N, $\mathrm{F}$. 
pendant $105 \mathrm{j}$, puis se stabilisent. Les populations dénombrées dans les lots $N$ et $F$ varient davantage. Un écart entre effectifs des lots $S$ et $F$ est noté en début d'expérience (fig 3d).

La proportion de Pseudomonas fluorescents dans la population bactérienne totale est plus élevée (principalement aux temps 10,30 et 154 j) dans le lot le plus arrosé $(F)$ que pour les 2 autres traitements (fig 4a).

Les populations d'actinomycètes, en pourcentage de la flore bactérienne totale, diminuent jusqu'au temps $30 \mathrm{j}$ pour les 3 traitements. Les effectifs du lot $F$ continuent à baisser pour se maintenir à un niveau très faible à partir de $45 \mathrm{j}$. Par contre, dans les deux autres lots, les populations augmentent (fig 4b).

Seuls sont représentés (fig 5) les Pseudomonas fortement chélatants (CMI de 32 à > 64 ppm) regroupés dans une classe unique ( $\geq 32 \mathrm{ppm}$ ) afin de faire ressortir leur comportement d'ensemble. Après $30 \mathrm{j}$ de traitements hydriques, ils sont moins nombreux qu'au temps 0 et aucune différence n'est observée entre traitements. Après 154 j, par contre, la répartition diffère selon le régime hydrique. Le pourcentage, très faible dans le lot $\mathrm{S}$, augmente avec la quantité d'eau apportée. Toutefois la différence n'est pas significative entre les traitements $\mathrm{N}$ et $\mathrm{F}$.

Les effectifs calculés à partir des dénombrements bactériens font apparaître, à $154 \mathrm{j}$, un
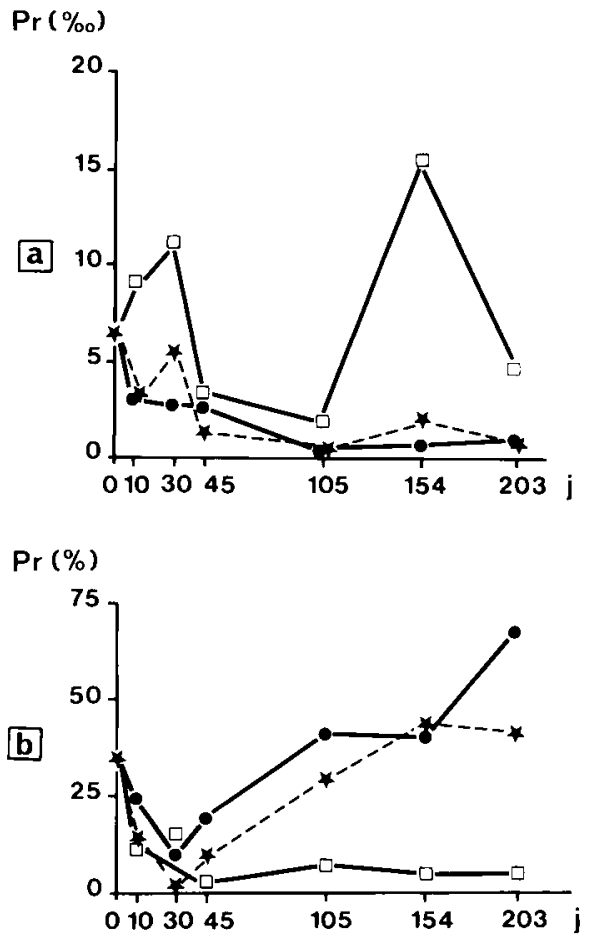

Fig. 4. Influence du régime hydrique sur la proportion $(\mathrm{Pr})$ de micro-organismes. a Pseudomonas fluorescents (en \% de la flore bactérienne totale). b actinomycètes (en \% de la flore bactérienne totale) $j \mathrm{j}=$ id fig $2 ; \bullet S, \star N, \square F$.

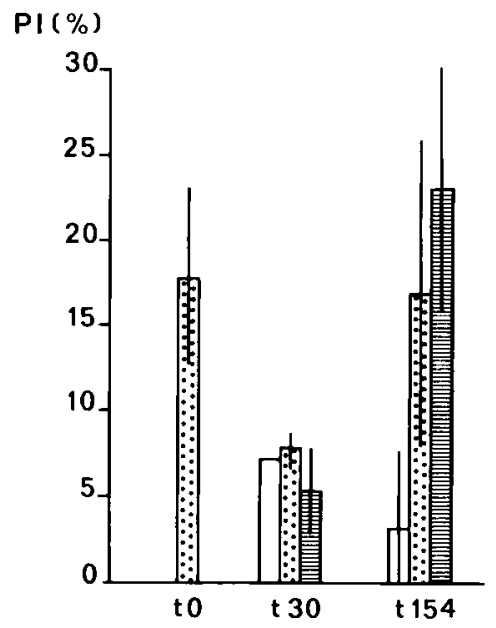

Fig. 5. Répartition des Pseudomonas à fort pouvoir chélatant ( $\geq 32 \mathrm{ppm}$ ) en fonction du régime hydrique, résultats exprimés en $\%$ des isolats (PI). $\square \mathrm{s}$, 䨨 $\mathrm{N}$, $\mathrm{F}$.

faible pouvoir chélatant total dans le lot $S$ par rapport à celui observé dans les 2 autres traitements (fig 6).

La distribution taxonomique (fig 7) montre qu'au temps 0 , l'essentiel de la population du rhizoplan est constitué par les espèces $P$ fluorescens et putida, présentes à des taux équivalents (respectivement 37,5 et $42,8 \%$ de l'effectif total). Les populations de $P$ fluorescens varient peu au cours du temps dans le lot N. Par contre, elles baissent dans les 2 autres lots, progressivement pour $F$, et avec un pic intermédiaire à $30 \mathrm{j}$ pour le lot le plus sec (S). Les effectifs de $P$ putida augmentent en fonction du temps quel que soit le régime hydrique, avec un effet plus marqué dans le lot le plus arrosé (F) à 154 j.

La répartition du pouvoir chélatant à l'intérieur de chaque taxon (fig 8) montre que ce sont essentiellement les espèces fluorescens et putida qui sont représentées au niveau des fortes CMI ( $\geq 32$ ppm) au temps 0 . Après 154 j de traitement, les plus fortes capacités chélatantes sont liées à l'espèce putida. A cette date, des

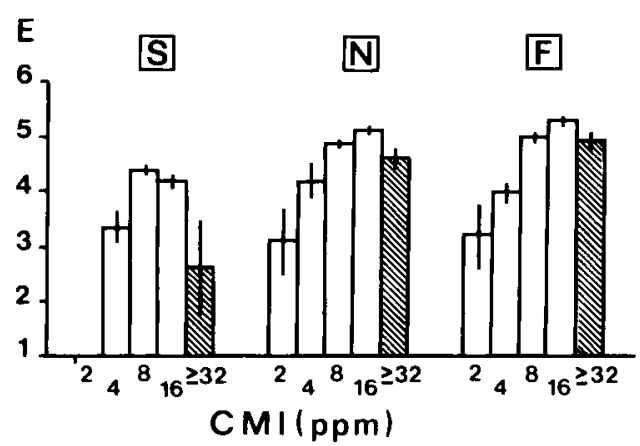

Fig. 6. Effet du régime hydrique sur le pouvoir chélatant des Pseudomonas isolés à 154 jours. $\mathrm{E}=$ effectifs calculés (log $\mathrm{CFU} / \mathrm{g} \mathrm{MS}$ ) pour chaque concentration inhibitrice (CMI) d'hydroxy-8-quinoléine. 


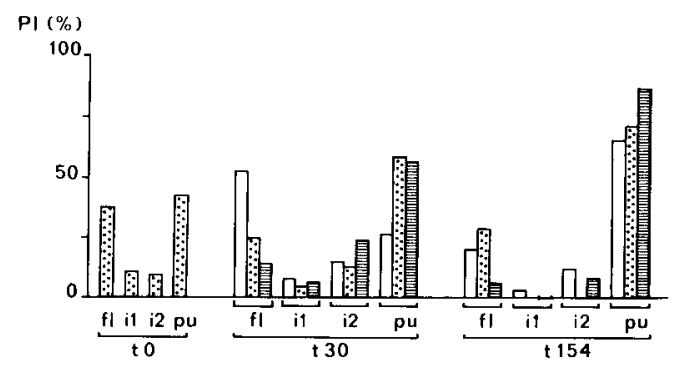

Fig. 7. Répartition taxonomique des Pseudomonas selon le traitement hydrique. Résultats exprimés en \% des isolats $(P I)$ pour chaque taxon. $\mathrm{fl}=P$ fluorescens, $\mathrm{pu}=P$ putida, $\mathrm{i}_{1}$ et $\mathrm{i}_{2}=$ groupes intermédiaires. $\square \mathrm{S}$, 四 N, 䁬 F.

germes à fort pouvoir sont aussi décelés dans le groupe $\mathrm{i}_{2}$ isolé du lot $\mathrm{F}$.

\section{Comportement des plantes}

En ce qui concerne le système aérien, après 5 mois de traitement, les plantes ont un nombre de tiges identique dans les 3 lots (fig 9a). La longueur du rameau de l'année de la pousse principale est comparable pour les noisetiers soumis à un régime sec ou normal, mais supérieure chez ceux qui ont subi une irrigation forcée (fig 9b). Un résultat semblable est observé en totalisant l'en-
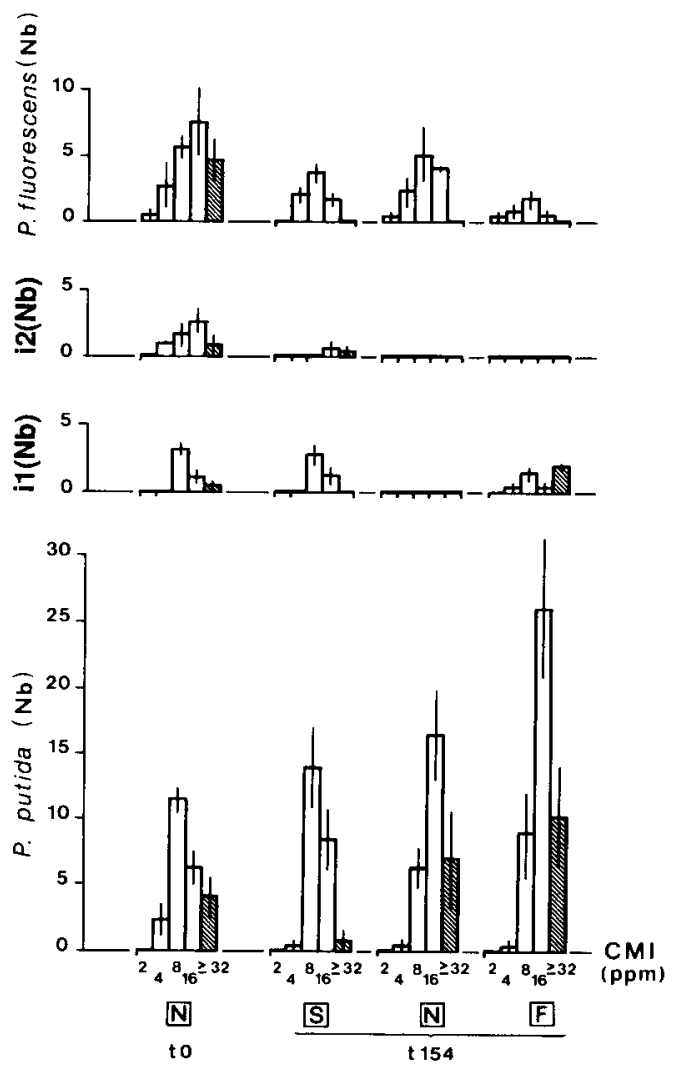

Fig. 8. Pouvoir chélatant des Pseudomonas selon le taxon et le traitement hydrique. Résultats exprimés en nombre d'isolats $(\mathrm{Nb})$ pour chaque concentration inhibitrice (CMI) d'hydroxy-8-quinoléine.
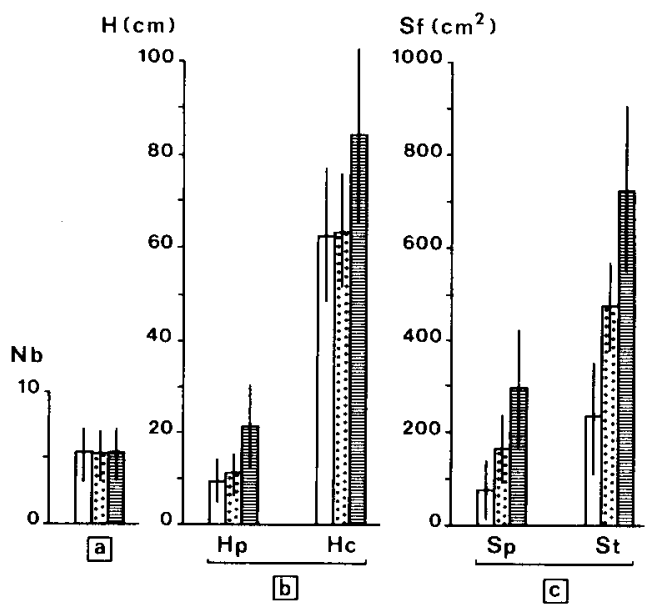

Fig. 9. Développement du système aérien selon les régimes hydriques. a nombre total de tiges (Nb) par plante; b hauteur $(H)$ des tiges, $\mathrm{Hp}=$ pousse de l'année de la tige principale, $\mathrm{Hc}=$ cumul des longueurs de tiges/plante; C. surface foliaire $(\mathrm{Sf})$ de la pousse de l'année $(\mathrm{Sp})$ et totale (St). $\mathrm{S}$, 圈 N, 罾 F.

semble des longueurs de tiges. La surface foliaire est directement corrélée à la quantité d'eau apportée (fig 9c), qu'il s'agisse de la surface foliaire totale ou de la surface foliaire développée au cours de l'essai (pousse de l'année).

\section{Mycorhization et système racinaire}

Des conditions de sécheresse (S) produisent un effet très marqué sur le système racinaire. Les jeunes racines sont presque inexistantes, aucune progression mycélienne n'a eu lieu depuis la plantation, mais les mycorhizes détectées au temps 0 se sont conservées, certaines très mélanisées, d'autres plus jeunes, turgescentes. Le régime normal $(\mathrm{N})$ a permis non seulement la conservation de la mycorhization existante au temps 0 , mais sa progression sur les jeunes racines, assez nombreuses dans ce traitement. Par contre, une forte irrigation $(F)$ a entraîné la régression de la mycorhization par la truffe dans la partie la plus âgée du système racinaire, et une faible colonisation des jeunes racines (fig 10). Dans ce lot, le système racinaire a un développement particulièrement intense et les racines occupent tout le volume du pot. Les jeunes racines sont très longues et très ramifiées.

Les mycorhizes de $T$ melanosporum portent des spinules en général bien réparties et nombreuses dans le lot $\mathrm{N}$, moins nombreuses ou groupées à l'apex dans le lot $\mathrm{S}$, et rares dans le lot $F$ : le développement mycélien hors de la mycorhize sensu stricto est faible dans le sol très irrigué. 


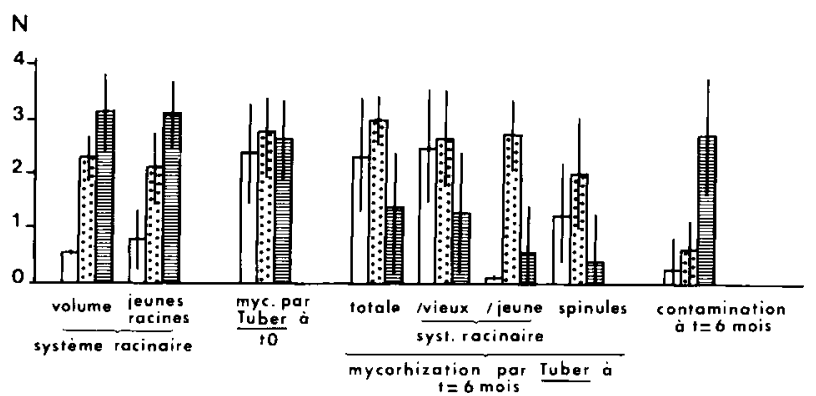

Fig. 10. Influence du régime hydrique sur le développement du système racinaire et sur la mycorhization. $\mathrm{N}=$ note (cf

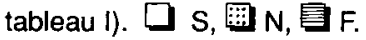

Une estimation de la contamination naturelle par d'autres mycorhiziens (toutes espèces confondues) montre une faible réceptivité des noisetiers appartenant aux lots sec et normal, et au contraire une très forte colonisation des plantes soumises à une irrigation forcée (fig 10). Mis à part 3 cas de contamination par des mycéliums sans caractéristique particulière, les compétiteurs peuvent être classés en trois types :

- B : mycélium de basidiomycète, avec nombreux branchements et nombreuses anses, formant un manteau en puzzle ;

- $G$ : très gros mycélium, roux foncé, sans anse, formant un manteau à très gros polygones irréguliers ;

- GR : mycélium granuleux, sans anse, branché à angles droits, formant un manteau en polygone (mycélium peu abondant, contrairement à celui des mycorhizes de type $A D$, d'aspect voisin, décrites par Giraud, 1988).

Dans le lot sec, seuls 2 noisetiers ont été contaminés, l'un par GR, l'autre faiblement par G. Aucune contamination n'a été détectée dans les 10 autres plantes contrôlées. Dans le lot normal, 9 noisetiers sur 12 sont contaminés, mais ils le sont en général faiblement. Le compétiteur majeur, par son taux de mycorhization (indice $\geq 2$ ) et sa fréquence (6 plants), appartient à la classe des basidiomycètes (type $B$ ). Les types $G$ et GR ne sont détectés qu'à l'état de trace (1 ou 2 mycorhizes) et respectivement sur 2 et 4 plantes. En régime détrempé, tous les noisetiers sont contaminés et les 3 types de compétiteurs sont présents, à des taux variables mais en général élevés. Leur fréquence est à peu près identique : 6 plantes contaminées par G, 7 par GR et 8 par B.

\section{DISCUSSION}

L'expérience décrite ci-dessus a permis d'analyser l'évolution de l'association noisetier-truffe en fonction du régime hydrique.
Le principal résultat concerne le comportement du Tuber et l'évolution de l'intensité de la mycorhization. Le taux de mycorhizes toutes espèces confondues ( $T$ melanosporum + contaminants) est sensiblement le même dans les 3 traitements, mais l'intensité de la mycorhization par la truffe varie selon les traitements hydriques. Un régime sec bloque le développement racinaire mais n'élimine pas le champignon. La mycorhization initiale est maintenue et l'organe mixte peut être considéré comme un site de résistance à la sécheresse. La densité du mycélium extramycorhizes (spinules) indique une augmentation de la surface d'échanges avec le sol. A l'opposé, un apport d'eau important réduit ou supprime ces hyphes externes et modifie profondément l'architecture du système racinaire. La colonisation des nombreuses racines jeunes par la truffe cesse et l'excès d'eau semble même détruire la mycorhization pré-établie. Dans ces conditions, la flore mycorhizienne compétitrice prolifère et remplace le Tuber sur les racines en développement; la réceptivité des racines à toute mycorhization n'est donc pas altérée. On peut penser que le maintien d'une trop forte irrigation $>>100$ $\mathrm{mm} / \mathrm{mois}$ ) ait, à terme de 2 ou 3 ans, un effet néfaste sur la production. Cette observation est compatible avec celle de Verlhac (in Giraud, 1988) réalisée au champ, et peut s'expliquer par un accroissement de la compétition. La truffe $T$ melanosporum demeure une espèce adaptée à des écosystèmes à faible disponibilité en eau, ce qui fixe des limites aux pratiques de forçage par irrigation. II est clair aussi qu'une irrigation moyenne (équivalente à $50-60 \mathrm{~mm} / \mathrm{mois}$ pour le sol de Dordogne) a un effet positif.

Un des buts de l'expérience était de suivre parallèlement l'évolution des populations bactériennes du mycorhizoplan. Contrairement aux observations de terrain (Olivier, Mamoun, 1988) les variations d'humidité ont peu modifié les niveaux de populations de Pseudomonas. Deux paramètres expérimentaux peuvent expliquer en partie cette différence. D'abord des températures élevées $\left(>30^{\circ} \mathrm{C}\right)$ ont régné dans le sol en été ; ces températures sont décrites par Loper et Schroth (1986) comme néfastes aux Pseudomonas en milieu carencé en fer. Ensuite, peuvent intervenir l'origine et la nature des Pseudomonas, introduits dans la terre stérilisée par le plant mycorhizé donc sélectionnés sur les racines dans un terreau de pépinière et non dans un sol de truffière. Cet inconvénient est difficilement contournable, car tout apport d'une flore de truffière (sol non stérilisé) se traduit, dans les conditions de serre ; par la prolifération de Scleroderma sp ou autres mycorhiziens compétiteurs, ce qui souligne la fragilité de la symbiose artificielle. 
On peut s'interroger sur la pérennité de cette association en sol de truffière, lorsque le potentiel de compétition est trop élevé, et qu'un apport d'eau destiné à assurer la reprise des plants est trop fort. Cela peut expliquer en partie les pourcentages variables d'arbres producteurs (de 1 à $82 \%)$ relevés en truffières par Chevalier (1983), un autre facteur intervenant, à savoir l'hétérogénéité génétique des plantes (Guinberteau et al, 1989). L'expérience a cependant permis de noter un accroissement des populations de Pseudomonas si l'eau augmente lorsque la température n'est pas limitante. L'inverse est observé pour les actinomycètes. On constate aussi que le pouvoir à chélater le fer des bactéries isolées augmente avec la teneur en eau, et par conséquent avec la disponibilité en fer ferreux, dans des conditions où la compétition s'accroît (plantes plus actives, flore fongique diversifiée). On retrouve cependant la précédente observation en truffière, à savoir une certaine incompatibilité entre multiplication des Pseudomonas et expansion mycélienne de Tuber. On note aussi que les températures dans le sol $\left(30^{\circ} \mathrm{C}\right.$ en été) n'ont pas gêné la truffe. En rapprochant cette observation des données déjà publiées (Mamoun et al, 1989), on peut affirmer que le mycélium de truffe est actif du printemps à l'automne, c'est-à-dire lorsque la température du sol dépasse $8{ }^{\circ} \mathrm{C}$ et que l'humidité est inférieure à un certain seuil fonction, entre autres, de la nature du sol.

Afin de poursuivre cette étude, il ressort de l'ensemble de ces observations la nécessité de travailler sur un végétal cloné, et de maîtriser les conditions d'hygrométrie et de température du sol. La production de clones de noisetiers (Guinberteau et al, 1989) va permettre de résoudre les problèmes d'hétérogénéité liés au matériel végétal. Des apports de bactéries connues (taxon, pouvoir chélatant) ou de flore fongique compétitrice (autre truffe, par exemple) sont en cours sur des plants mycorhizés par T melanosporum pour préciser les interactions entre cette truffe et la microflore indigène.

\section{REMERCIEMENTS}

Ce travail a bénéficié d'une subvention européenne dans le cadre du Plan d'Intégration Méditerranéenne et du projet «relance de la trufficulture en Aquitaine».

\section{RÉFÉRENCES}

Cailleux M (1982) Biologie de la truffe - différentes espèces. CR Stage CTIFL, 2, Ballendran, 8-9 mars 1982
Chevalier G (1983) Production de truffes à partir de plants mycorhizés selon le procédé INRA: premiers résultats. Bull FNPT, 6, novembre 1983, 3350

Giraud M (1988) Prélèvement et analyse de mycorhizes. Bull FNPT, 10, juillet 1988, 49-63

Guinberteau J, Salesses G, Olivier JM, Poitou N (1989) Mycorhization de vitroplants de noisetiers clonés. $2^{e}$ Cong Intern truffe, Spoleto (sous presse)

Hohnadel D, Meyer JM (1986) Pyoverdine - facilitated iron uptake among fluorescent Pseudomonads. In : Iron, Siderophores and Plant diseases. (Swinburne, TR ed), Nato-Plenum, Londres, pp 119-129

Lemanceau P (1988) Réceptivité des sols aux fusarioses vasculaires : étude critique des théories proposées. Thèse Doct d'Univ, Lyon I, $95 \mathrm{p}$

Lemanceau P, Samson R, Alabouvette C (1988) Recherches sur la résistance des sols aux maladies. XV : Comparaison des populations de Pseudomonas fluorescents dans un sol résistant et un sol sensible aux fusarioses vasculaires. Agronomie 8, 243-249

Le Tacon F, Delmas J, Gleyze R, Bouchard D (1982) Influence du régime hydrique du sol et de la fertilisation sur la fructification de la truffe noire du Périgord. (Tuber melanosporum Vitt) dans le Sud-Est de la France. Acta Oecologia, Oecol Appl, 3, 291 . 306

Lindsay WL (1974) Role of chelation in micronutrient availability. In: The Plant root and its environment (Corson EW ed). University Press, Virginia, Charlottesville, pp 507-527

Loper JE, Schroth MN (1986) Importance of siderophores in microbial interactions in the rhizosphere. In : Iron, Siderophores and Plant diseases (Swinburne TR ed). Nato-Plenum, Londres, pp 85-98

Mamoun M, Olivier JM (1989) Dynamique des populations fongiques et bactériennes de la rhizosphère des noisetiers truffiers. If : Chélation du fer et répartition taxonomique chez les Pseudomonas fluorescents. Agronomie, 9, 345-351

Mamoun M, Olivier JM, Guinberteau J (1989) Milieux de culture et croissance mycélienne de la truffe. $2^{e}$ Cong Intern Truffe, Spoleto (sous presse)

Olivier JM, Mamoun M (1988) Dynamique des populations fongiques et bactériennes de la rhizosphère des noisetiers truffiers. I : relation avec le statut hydrique du sol. Agronomie, 8, 711-717

Palleroni NJ (1984) Pseudomonadaceae. In : Bergey's Manual of Systematic Bacteriology, 3rd ed, 1, (Williams, Wilkins, ed). Baltimore, Londres, pp. 141 . 199

Römheld V (1987) Existence of two different strategies for the acquisition of iron in higher plants. In: Iron Transport in Microbes, Plants and Animals. (Winkelmann G, Vand der Helm D, Neilands JB, ed) VCH, Allemagne, pp. 353-373

Scher FM (1986) Biological control of Fusarium wilts by Pseudomonas putida and its enhancement by Eddha. In : Iron, Siderophores and Plant diseases. (Swinburne, TR ed). Nato-Plenum, Londres, pp. 109-117

Szanislo PJ, Powell PE, Reid CPP, Cline GR (1981) Production of hydroxamate siderophore iron chelators by ectomycorrhizal fungi. Mycologia, 73, 1 1581174 